Theoretical Physics, Astrophysics and Cosmology

Vol. 8, No 1, p. 16 - 23, v1, 15 April 2013

Online: TPAC: 4488-029 v1, 15 April 2013
ISSN 1819-9135; EISSN 1991-3919

(C) 2013 CTPA. All rights reserved. DOI: 10.9751/TPAC.4488-029

\title{
The frequency and intensity stasis effects for radiation crossed galaxy clusters. 2. Isotropy and anisotropy of CMB.
}

\author{
Zahid Zakir $^{1}$
}

\begin{abstract}
In the previous two papers it has been studied a new class of general relativistic effects the frequency and intensity stasis of radiation crossing gravitationally-bound regions (GBRs), such as galaxy clusters, due to switching out from the cosmological expansion flow. In the present paper the theory of stasis effects is applied to cosmic microwave background (CMB). For a part of CMB crossing a cluster these effects lead to the stasis of its temperature and «heating» w.r.t. a normally expanded flow outside the cluster. As a result, instead of former paradigm about almost sterile propagation of CMB from the recombination epoch, in fact, general relativity and cosmological principle lead to the opposite picture. Mixing of flows of relic photons, repitedly isolated many times from expansion flow in GBRs along their path, sufficienly changes the physical characteristics of CMB, particularly, leads to its isotropy and loss of earlier times perturbations. The observing anisotropy follows from the stasis effects at crossing of multiple layers of clusters at our nearest environment.
\end{abstract}

PACS: 98.80.Es, 97.60.Bw, 98.65.Cw, 98.62.Py, 98.62.Ck, 98.62.Qz, 98.80.Jk, 98.70.Vc

Key words: cosmology, galaxy clusters, gravitation, background radiation, anisotropy

\section{Content}

Introduction .16

1. The CMB temperature stasis effect in galaxy clusters ..........................................................17

2. Anisotropy of CMB due to the stasis effects in nearest clusters ............................................18

3. Isotropy of CMB due to temperature stasis in distant regions of inhomogeneities..................19

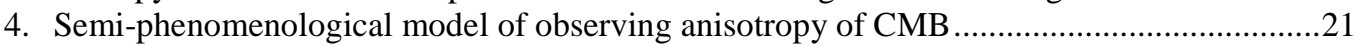

References.

\section{Introduction}

In the previous papers [1,2], where the stasis effects following from general relativity (GR) for frequency and intensity of radiation crossing the gravitationally-bound regions (GBRs), largest of which are galaxy clusters, have been studied, already it has been noted that such stasis of radiation temperature can generate quasi-periodic peaks in angular distribution of temperature and polarisation of CMB. Estimations showed that galaxy clusters at last stage of flow's path should contribute essentially to the observing anisotropy of CMB.

In the present paper it will be shown that this latest time contribution not only is essential, but it is a basic mechanism generating the observing anisotropy of CMB. It related also with the fact that perturbations of CMB due to the temperature stasis in GBRs

\footnotetext{
${ }^{1}$ Centre for Theoretical Physics and Astrophyics, Tashkent, Uzbekistan; zahidzakir@theor-phys.org
} 
appear as of the same order, as at the recombination epoch, so all of them become smoothed due to repeated perturbations and mixings at propagation of the relic flow. This explains the high isotropy of the relic flow incoming into our region of inhomogeneity at radius $<150$ Mps.

Notice, that if at the Sachs-Wolfe effect, related by the gravitational shift of frequencies, at leaving a cluster the relic flow becomes colder, at the stasis effects the leaving flow becomes heater than uncrossed flow. But, unlike other known effect of heating - the Zeldovich-Sunyaev effect related by interaction of CMB with matter of the cluster, - the stasis effects, being a reverse side of the cosmological expansion, are universal, independent on the matter content and are determined only by size and configuration of GBRs.

In sections 1-3 the theory of stasis effects for CMB and formulas for corrections are presented and in section 4 the phenomenological model of the observing anisotropy of CMB following from the new mechanism of its generation is proposed.

\section{The CMB temperature stasis effect in galaxy clusters}

In the first part of the present paper [2] it has been obtained a relation between the observing redshift $Z$ and the "effective" redshift $\bar{Z}$ which would be observed in fully homogeneous world (for the standard closed model of GR without dark energy). For CMB the ratio of temperature at earlier epoch $T_{e}$ to the present $T_{r}$ is determined by the ratio of observing wavelength $\lambda_{r}$ to wavelength $\lambda_{e}$ at that epoch:

$$
\frac{T_{e}}{T_{r}}=\frac{\lambda_{r}}{\lambda_{e}}=1+z
$$

At the same time, the ratio of the present cosmological scale factors $a_{0}$ and earlier epoch $a_{\bar{z}}$ determine normal («effective») redshift $\bar{z}$ and the ratio of temperatures $\overline{T_{e}}, \bar{T}_{r}$ corresponding to a fully homogeneous world:

$$
\frac{\bar{T}_{e}}{\bar{T}_{r}}=\frac{a_{0}}{a_{\bar{z}}}=1+\bar{Z} .
$$

In the homogeneous world these two kinds of temperatures are equal and in the Friedmann model the equation for decreasing of the CMB temperature looks like:

$$
\frac{d T}{T}=\frac{d a}{a} .
$$

However, in the presence of GBRs these definitions are inequivalent since $\bar{T}<T$ , i.e. the stasis of temperature of the flow in GBRs leads to relative heating. The theory of this effect can be formulated similar to [2], but only instead of wavelengths, redshifts and luminosities it is enough to consider only the temperature of CMB.

Main changing in the rate of cooling of CMB is that at crossing an inter-cluster distance $\Delta l_{c}$ temperature of the flow changes not everywhere, but only out of $\Delta l_{g}$, i.e. only in the fraction $(1-f) \Delta l_{c}$ of the full distance, where $f \equiv \Delta l_{g} / \Delta l_{c}$. Accordingly, temperature decreasing $\Delta T$ happens not on all interval of the scale factor addition $\Delta a$, but only in its part equal to $\Delta a \cdot(1-f)$ and, instead of (3), we have a new equation: 


$$
\frac{d T}{T}=\frac{d a}{a}(1-f) .
$$

With the account of expression for $f[1]$ :

$$
f=\frac{\Delta l_{g}}{\Delta l_{c}}=\frac{\Delta l_{g}}{\Delta l_{c 0}} \frac{a_{0}}{a}=f_{0} \frac{a_{0} H_{0}^{2 / 3}}{a H^{2 / 3}},
$$

the equation (4) takes the form:

$$
\frac{d T}{T}=\frac{d a}{a}-\bar{w} \frac{d a}{a^{2} H^{2 / 3}} .
$$

Here $\bar{w}=f_{0} a_{0} H_{0}^{2 / 3}$ and $H=\dot{a} / a$ is the Hubble parameter which is model dependent.

In the standard closed model of GR (without dark energy) with the maximal radius of expansion $a_{m}=2 G M / c^{2}$, where $M=(4 \pi / 3) \rho_{0} a_{0}^{3}$, we have:

$$
H=\frac{a_{m}^{1 / 2} c}{a^{3 / 2}}\left(1-a / a_{m}\right)^{1 / 2}
$$

and the equation (6) turns to:

$$
\frac{d T}{T}=\frac{d a}{a}-w \frac{d a}{a\left(1-a / a_{m}\right)^{1 / 3}},
$$

where $w=f_{0} y_{0}, y_{0}=(1-b)^{1 / 3}$ and $b=a_{0} / a_{m}$. Integration of this equation gives required connection between $T$ and $\bar{T}$ :

$$
\frac{T_{e}}{T_{r}}=\frac{\bar{T}_{e}}{\bar{T}_{r}} \cdot \frac{\exp \left\{3^{1 / 2} w \cdot \arctan \left[\left(1+2 y_{z}\right) / 3^{1 / 2}\right]\right\}}{G \cdot\left[1+3 y_{z} /\left(1-y_{z}\right)^{2}\right]^{w / 2}}
$$

where:

$$
\begin{gathered}
y_{z}=\left(1-a_{z} / a_{m}\right)^{1 / 3}=\left[1-b \bar{T}_{r} / \bar{T}_{e}\right]^{1 / 3}, \\
G=\frac{\exp \left\{3^{1 / 2} w \cdot \arctan \left[\left(1+2 y_{0}\right) / 3^{1 / 2}\right]\right\}}{\left[1+3 y_{0} /\left(1-y_{0}\right)^{2}\right]^{w / 2}} .
\end{gathered}
$$

For expression $\bar{T}$ through $T$ it is necessary to invert Eq. (9) that analytically is very difficult. But in two extreme cases when initial and final temperatures differs very little (Section 2) or very large (Section 3), the formula (9) simplifies and can be inverted.

\section{Anisotropy of CMB due to the stasis effects in nearest clusters}

Let us at first estimate a «heating» of CMB at crossing one of closest galaxy clusters. Time of crossing by radiation of the cluster with radius of GBR at least $\Delta l_{g 0} \sim 2 \mathrm{Mps}$ is about $6.5 \mathrm{mln}$ years which is approximately $5 \cdot 10^{-5}$ times of $13.5 \mathrm{bln}$ years, the time close to the recombination epoch. For all time after recombination the CMB temperature has decreased about $10^{3}$ times, but almost all changing was occurred on first 3.5 bln years, whereas during last 10 bln years CMB temperature has decreased only to few $10^{1} \mathrm{~K}$, i.e. lowered averagely to few $10^{-4} \mathrm{~K}$ each mln year. But most part of this changing occurred again in earlier half of this time due to larger velocity of expansion, whereas at present it makes few $10^{-5} \mathrm{~K}$ per mln year.

However, the relic flow between clusters also is expanded unideally since restoring of the full cosmological receding velocity happens only at distances few $\Delta l_{g 0}$, so this part of the flow is «heated» too, although smaller than in the clusters. As a result, 
«heating» of the flow crossed clusters will be less w.r.t. uncrossed flow and will be of order few $10^{-5} \mathrm{~K}$.

Let us turn to more consistent derivation of these conclusions. At a small changing of temperature $\Delta \bar{T} \ll \bar{T}_{r}$ we can take series of right hand side of (9), after which the formula simplifies and gives:

$$
\begin{aligned}
\frac{\Delta T}{T_{r}} & \simeq\left(1-f_{0}\right) \frac{\Delta \bar{T}}{\bar{T}_{r}}+Q \cdot\left(\frac{\Delta \bar{T}}{\bar{T}_{r}}\right)^{2}, \\
Q & =\frac{\alpha f_{0}}{6}\left(\frac{1}{1-b}-4+3 f_{0}\right) .
\end{aligned}
$$

where $\alpha$ is the correction coefficient selected for correspondence of the former precise and latter simplified versions of the formula.

Further for each combination of parameters $b, f_{0}$ we find such $\alpha$ which gives in Eq. (12) a curve close to Eq. (9) and then we obtain the required formula:

$$
\begin{aligned}
\frac{\Delta \bar{T}}{\bar{T}_{r}} & \simeq \frac{1-f_{0}}{2 Q}\left[1-\sqrt{1-4 Q \cdot \Delta T / T_{r}\left(1-f_{0}\right)^{2}}\right] \simeq \\
& \simeq \frac{1}{1-f} \frac{\Delta T}{T_{r}}\left(1+\frac{Q}{(1-f)^{2}} \frac{\Delta T}{T_{r}}\right) .
\end{aligned}
$$

Thus, we see that heating of CMB in galaxy clusters due to isolation from the expansion flow represents very sufficient effect. In ideal case it has the same order as a redshift stasis for supernovae which was considered in [1,2]. However, since the CMB temperature is determined by averaging on all sky (or on large enough regions), real difference of temperatures of whole background and a flow from a cluster becomes much less and appears as few $10^{-5} \mathrm{~K}$.

\section{Isotropy of CMB due to temperature stasis in distant regions of inhomogeneities}

The considered model example shows that though effect of CMB temperature stasis in clusters several times less than the main phenomenon - cooling of the relic flow outside clusters due to cosmological expansion - but it is of the same order and in few orders exceeds all other effects for CMB, making almost same dominating influence on main properties of $\mathrm{CMB}$, as the expansion.

Moreover, the stasis effect in few layers of nearest to us galaxy clusters has the same order of magnitude $\left(10^{-5}\right)$, as perturbations of CMB at recombination epoch.

At first view this result looks rather strange since the relic flow has crossed hundreds such regions of inhomogeneity, as our environment $<150 \mathrm{Mps}$, which earlier epochs have ten times smaller size. If in each of them the flow experienced the same perturbations, as at the recombination epoch, the initial information there should be fully lost since all perturbations from enough distant regions of inhomogeneity will mix up and the flow should form some highly isotropic background.

In fact this is not inconsistency, but a paradigm shift, namely, from the stasis effects and the cosmological principle follows two conclusions of prime importance, opposite to the current paradigm. The first one is: 
(A) all perturbations of CMB arising at distances exceeding $150 \mathrm{Mps}$ from us, will reach us almost completely smoothed due to the stasis effects in large number of GBRs (clusters of protogalaxies and galaxies) and it seems that the relic flow incoming into our region of inhomoheneity is isotropic with high accuracy $\left(\sim 10^{-6} \mathrm{~K}\right)$.

Thus, if in a ball of radius 150 Mps around us there would be a void or homogeneous media, the arriving relic flow would be observed as almost isotropic with temperature anisotropy no more $10^{-6}$.

For the expressing $\bar{T}$ through $T$ we need in inverting Eq. (9), which can be done only by some approximation, and here we consider Eq. (9) in asymptotes:

$$
\begin{aligned}
& \frac{T_{e}}{T_{r}}=F \cdot\left(\frac{\bar{T}_{e}}{\bar{T}_{r}}\right)^{1-w}, \quad \bar{T}_{e} \gg \bar{T}_{r} \\
& F=27^{-w / 2} b^{w} \exp \left\{4 \pi w / 3^{1 / 2}\right\} / G .
\end{aligned}
$$

This asymptotes is sensitive to a choice of the parameters of the model $b$ and $f_{0}$. At values $b=0.3, f_{0}=0.15$ Eq.(15) turn to:

$$
\frac{T_{e}}{T_{r}} \approx 0.44 \frac{\bar{T}_{e}}{\bar{T}_{r}} .
$$

From this relation follows that at supposing the same values of both kind of temperatures at the recombination epoch $\bar{T}_{e} \approx T_{e}$, we find that to the observing temperature $T_{r} \simeq 2.72 \mathrm{~K}$ at present corresponds the unperturbed temperature

$$
\begin{aligned}
& \bar{T}_{r} \approx 0.44 \cdot T_{r} \approx 1.20 \mathrm{~K}, \\
& \Delta T \simeq 1.52 \mathrm{~K}
\end{aligned}
$$

where $\Delta T$ is heating of CMB due to the stasis effects in GBRs.

From considered in the first section of the theory of effect of stasis and above presented conclusion (A) if follows the second conclusion of prime importance

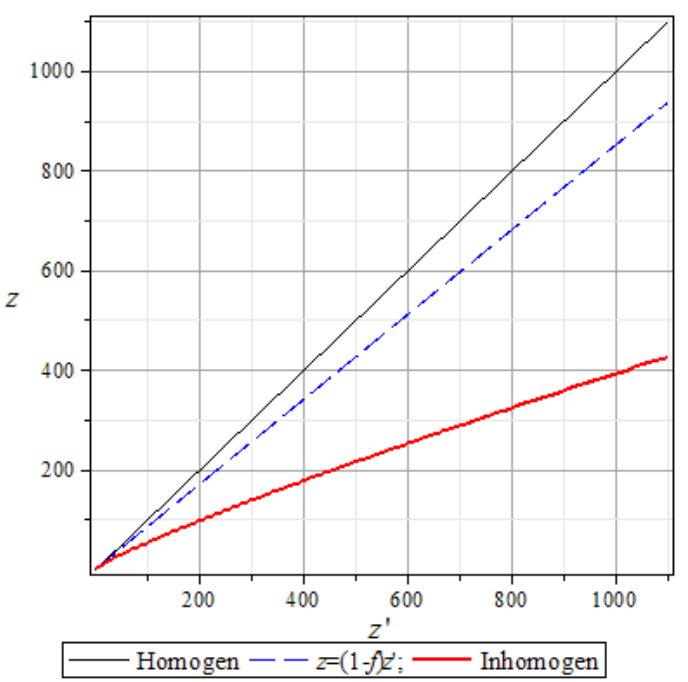

Fig. 1. Asymptotes of $z(\bar{z})$ up to $\bar{z}=1000$ at $f_{0}=0.15, b=0.3$ completely overturning the current paradigm:

(B) almost all observing perturbations of $C M B$ of the order $10^{-}$ ${ }^{5} \mathrm{~K}$ and more have arisen mainly in the inhomogeneity region around us of radius less that $150 \mathrm{Mps}$ due to the temperature stasis in few (6-10) layers of galaxy clusters.

This situation makes the problem of construction of the theory of observing anisotropy practically solvable by studying of our nearest environment up to $150 \mathrm{Mps}$, where practically all essential contributions are available to measuring with much more higher accuracy than for more distant regions. 


\section{Semi-phenomenological model of observing anisotropy of CMB}

Observing anisotropy of CMB, thus, mainly is generated by the nearest to us layers of clusters which are well studied. For this reason before formulating the consistent microscopic theory of anisotropy, a way to it will be better to begin with discussion of a simple working model in general expressing the new physical mechanism of this phenomenon described in the previous sections. Further how will change some conjectures of this simplified model, it is enough to play a role of a starting point for investigations on the new basis.

A mean distance between groups and clusters of galaxies exceeds size of their GBRs at least several times. Observing angular anisotropy of CMB temperature will be related mainly by the «heating» of the relic flow at its crossing through a number of overlapped clusters along a sight of view in comparison with the flow penetrated through this distance without crossing any cluster.

Largest of peaks in the power spectrum of angular anisotropy of temperature with $D_{l, 0}^{1 / 2} \simeq 75 \mu \mathrm{K}$ and angular number $l \simeq 220$ (Fig. 2 - 3) then it is natural to relate mainly with the unperturbed flow penetrated between clusters and for this reason which was cooled almost without stasis and appears as the coldest.

The subsequent peaks $D_{l, 1}^{1 / 2}, \ldots, D_{l, 6}^{1 / 2}$ or cavities $\tilde{D}_{l, 1}^{1 / 2}, \ldots, \tilde{D}_{l, 6}^{1 / 2}$ with the decreasing height we can relate by the flows which crossed averagely through one or multiple layers of clusters. Depending on a sign of deviation of temperature, and also, whether the temperatures are count - from temperature of the coldest peak or from the warmest peak, the interpretation will changes. Therefore, having left a problem of improvement of interpretation for further more consistent study, further we will obtain formulas both for peaks and cavities.

At crossing through some clusters rise in temperature w.r.t. the coldest first peak should be averagely multiple to the decrement of temperature in one cluster: $\Delta D_{l, n}^{1 / 2} \sim-n \cdot \delta T$ and, consequently, the square root of power spectrum $D_{l, n}^{1 / 2}$ should fall approximately equidistantly up to a value at large $l$. Further it is enough to consider only 6 layers of nearest clusters $(n=1,2, \ldots, 6)$, sufficiently perturbed CMB. Notice, that the disturbed flow at propagation between clusters is cooled lesser (with coefficient $p$ ), than the unperturbed flow penetrated between clusters, since here the expansion velocity does not completely recovered. Thus, for heights of peaks and cavities we obtain following simple formulas:

$$
\begin{aligned}
& D_{l, n}^{1 / 2} \simeq p \cdot D_{l, 0}^{1 / 2}-n \cdot \delta T, n>0 . \\
& \tilde{D}_{l, n}^{1 / 2} \simeq \tilde{p} \cdot \tilde{D}_{l, 0}^{1 / 2}-n \cdot \delta \tilde{T}, n>0 .
\end{aligned}
$$

The largest contribution to a flow crossed through one cluster give the nearest to us clusters of the first layer since their mean angular diameter $\Delta \theta_{1}$ is largest. The largest contribution to a flow crossed through two clusters give the clusters of the first and second layers and since angular diameter of the second layer clusters averagely twice less than in the first layer: $\Delta \theta_{2}=\Delta \theta_{1} / 2$, this flow's peak has larger angular number $l_{2}>l_{1}$. Similarly, in a flow crossed 6 clusters should contribute a cluster of the sixth layer, a mean angular diameter of which is $\Delta \theta_{6}=\Delta \theta_{1} / 6$. Thus, the number of partitions of sky 
in each layer also monotonically increases and we can take the linear approximation as simplest one. For positions of peaks then we obtain a semi-empirical formula:

$$
l_{n}=l_{0}+\Delta l \cdot n \simeq 220+300 \cdot n .
$$

The radiation flow which has crossed only one cluster will be warmer of uncrossed flow to $\delta T_{1}$ and the theoretical estimation gives $\delta T_{1} \sim 10^{-5} \mathrm{~K}$. Further we will consider this quantity as a parameter of the model for which from comparison with observations we obtain the values $\delta T_{1} \simeq 6.5 \mu \mathrm{K}$ (cavities) or $\delta T=7.5 \mu \mathrm{K}$ (peaks). In this flow, one times crossed a cluster, contribute flows from those parts of clusters of each layer which in a sight of view do not overlapped by other clusters neither in front, nor behind. This flow has in the sky larger recurrence than the uncrossed flow, so it corresponds to the first peak after the largest (cooled) one. The seventh peak contains contributions from all of 6 overlapped layers of clusters. For this reason its deviation from the CMB temperature for large $l$ will be minimal and this peak or cavity will be smallest.

Further, by using (18)-(19) we obtain from the observational data [3]: $\tilde{p}=0.79$, $\delta \tilde{T}=6.5 \mu \mathrm{K}$ for cavities (Fig. 2) and $p=0.69, \delta T=7.5 \mu \mathrm{K}$ for peaks (Fig. 3).

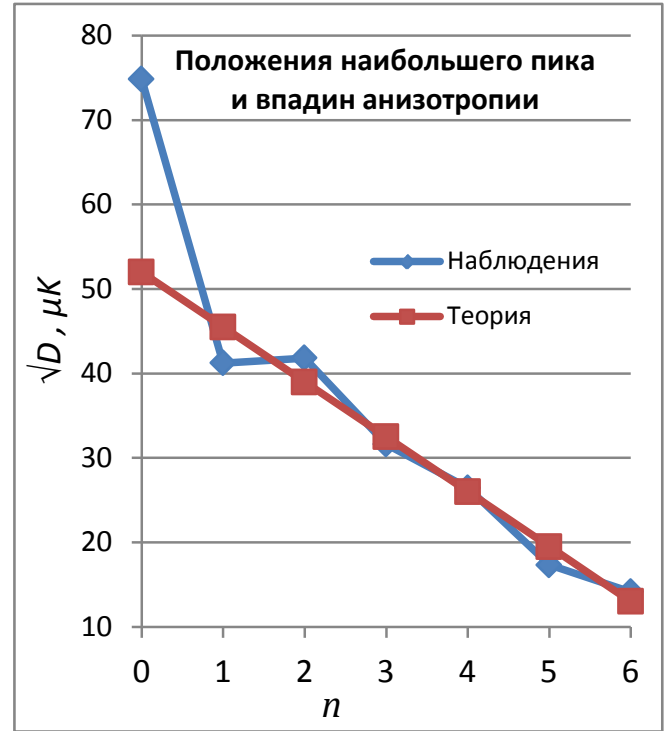

Fig.2 Positions of largest peak (with $n=0$, $l \approx 220$ ) and 6 cavities in the diagram of $\tilde{D}_{l, n}^{1 / 2}$.

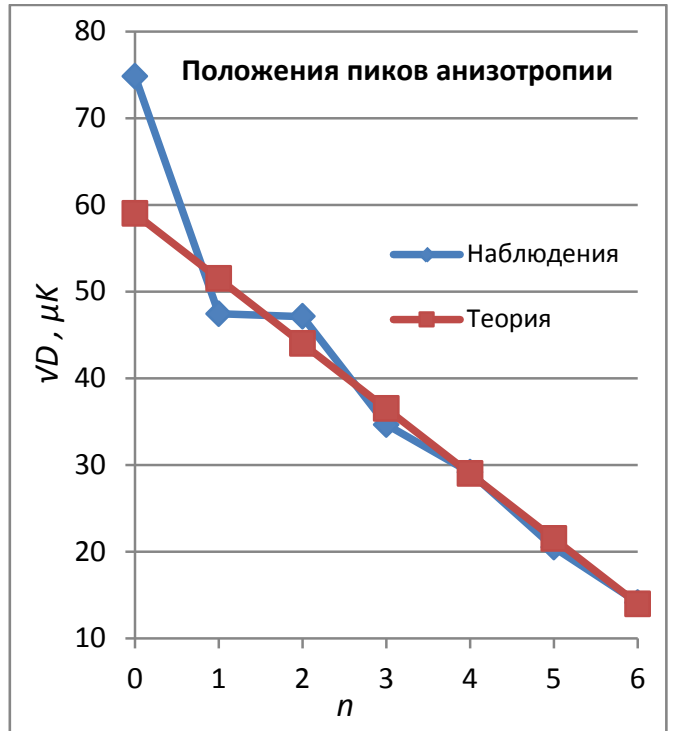

Fig.3 Positions of peaks in the diagram of $D_{l, n}^{1 / 2}$.

Width of peaks depend on different factors, such as diffusiveness of borders clusters, Doppler effect due to centre of mass velocity of clusters, etc., so they in principle can be calculated for each layer. For the present row estimations it is enough to use the Gauss character of these contributions.

Thus, in the framework of the considered working model the smallest anisotropy peak - the 7-th - we relate by the relic flow crossed through six clusters, and higher peaks we relate by flows crossed through smaller number of clusters. As the result, by determining the positions and heights of the smallest and highest peaks of anisotropy as the parameters, the model allows us to calculate in the first approximation positions and heights of the intermediate peaks, and then cavities too. 


\section{Conclusion}

Main features of above presented treatment of properties of CMB, including explanations of isotropy and anisotropy, are direct consequences of GR and cosmological principle, and in this sense are fundamental.

Some used approximations, numerical estimations or comparison with observations will be subject of more detail investigations and should be improved.

Further development of this treatment, most likely, will allow describing more complicate properties of $\mathrm{CMB}$, such as angular anisotropy of polarisation.

In this situation, if it will be confirmed that the new treatment allows revealing and explaining the basic properties of CMB by starting from general requirements of GR for radiation in GBRs, it becomes new obvious advantage of GR. This circumstance then will have value irrespective of how will vary at the further improvements the used simplified model.

\section{References}

1. Zakir Z. (2013) Theor. Phys., Astrophys.\& Cosmol. 8, 1, p. 1.

2. Zakir Z. (2013) Theor. Phys., Astrophys.\& Cosmol. 8, 1, p. 7.

3. Planck Collaboration (2013) Report XXIII, arXiv:1303.5083, 43 p. 